Original article

\title{
Phenolic contents, antioxidant activities and potential bioaccessibilities of industrial pomegranate nectar processing wastes
}

\author{
Ece Surek $^{1,2} *$ \& Dilara Nilufer-Erdil ${ }^{1}$ \\ 1 Department of Food Engineering, Faculty of Chemical and Metallurgical Engineering, Istanbul Technical University, 34469 Maslak, \\ Istanbul, Turkey \\ 2 Department of Food Engineering, Faculty of Engineering, Izmir Institute of Technology, 35430, Gulbahce, Urla, Izmir, Turkey
}

(Received 15 July 2015; Accepted in revised form 2 October 2015)

\begin{abstract}
Summary Antioxidant potential and bioaccessibility of co-products from industrial pasteurised pomegranate nectar (PN) processing such as peel (PP), press cake (PC) and precipitate after clarification (PAC) in comparison with raw material (arils) and final products (CON and PN) were determined. Total phenolic (TPC), flavonoid (TFC), anthocyanin (TAC), tannin contents (TTC) and antioxidant activity (TAA) were determined besides identifying major phenolics and investigating in vitro bioaccessibility after gastrointestinal (GI) digestion. PP showed the highest values, except for TAC. Phenolics (12.7-43.0\%) were found to be more stable than anthocyanins (0.6-2.1\%) after in vitro GI digestion. PAC was found to be a better source for anthocyanins than CON and also showed higher phenolic bioaccessibility $(28.8 \%)$ than PN $(19.6 \%)$. PC and PAC possessed as much TPC, TFC, TTC and TAA levels as CON, with some exceptions. Therefore, these results indicated that not only PP but also PC and PAC should be valorised as a good source for phenolics and anthocyanins.
\end{abstract}

Keywords Antioxidant activity, bioaccessibility, co-product, in vitro digestion, nectar, peel, pomegranate, precipitate, press cake, waste.

\section{Introduction}

Pomegranate (Punica granatum) is a potential antioxidant source, and each of its parts (peel, seeds, arils and inner peel) have valuable compounds providing several functional and medicinal properties to this fruit. It can act as antitumoral, antidiabetic, antihepatoxic and antimicrobial (Viuda-Martos et al., 2010). It consists of $60-67 \%$ arils and $33-40 \%$ peel (Adsule \& Patil, 1995). Juice can be produced from arils as well as the whole fruit (Adsule \& Patil, 1995). Pomegranate contains polyphenols such as flavonoids (flavonols, flavanols, anthocyanins), condensed tannins (proanthocyanidins), hydrolysable tannins (ellagitannins and gallotannins) and other phytochemicals such as organic and phenolic acids, sterols, fatty acids, triglycerides and alkaloids (Elfalleh et al., 2011). Pomegranate can be processed into many different products such as canned arils, dried arils, seeds, syrup, jam, sour sauce, concentrate and nectar in addition to its consumption as a fresh fruit (Adsule \& Patil, 1995).

*Correspondent: Fax: +90-232-7506196; e-mail: ecesurek@iyte.edu. $\operatorname{tr}$
As pomegranate cannot be grown at all seasons, it can be consumed for longer durations when postharvest treatments such as optimum cold storage, modified atmosphere packaging and controlled atmosphere are applied (Selcuk \& Erkan, 2015) or it is processed into different products. Due to the fact that bioavailability and antioxidant potential of pomegranate products should also be investigated. Although there are several studies focusing on the processing steps of pomegranate juice (Gil et al., 2000; Alper et al., 2005; Alighourchi et al., 2008; Turfan et al., 2011; Rinaldi et al., 2013) or nectar (Surek \& Nilufer-Erdil, 2014), there is limited research on investigating the potential antioxidant power or polyphenol contents of pomegranate co-products (Viuda-Martos et al., 2011), except peel. Polyphenol content, antioxidant activity or bioaccessibility of some industrial fruit juice processing wastes have been analysed by many researchers. According to Lee \& Wrolstad (2004), blueberry juice processing wastes showed high total anthocyanin and phenolic content. On the other hand, waste residues such as press cake had very low levels of the main anthocyanins during sour cherry nectar processing (Toydemir et al., 2013). Suzme et al. (2014) investigated 
effect of juice processing on polyphenols and antioxidant capacity of black carrot. There was no difference between raw material and press cake according to total flavonoid content and antioxidant capacity; however, press cake had higher total phenolic and anthocyanin content than raw material. There is limited research on potential bioavailability of pomegranate bioactive compounds (Perez-Vicente et al., 2002; Sengul et al., 2014; Xuan et al., 2014). Perez-Vicente et al. (2002) and Sengul et al. (2014) researched in vitro bioavailability of anthocyanins and total phenols of pomegranate juice and pomegranate fruit, respectively. Phenolics were found to be stable during gastric conditions; however, $29 \%$ of juice and $14 \%$ of fruit were available in the dialysed fraction (IN). Anthocyanins of pomegranate were lost in pancreatic digestion $(38 \%)$, but available (12\%) in IN (Sengul et al., 2014). In the research by Xuan et al. (2014), the loss of anthocyanins of pomegranate wine was low during gastric digestion; however, $25 \%$ of anthocyanins were available after gastrointestinal (GI) digestion.

In this study, the main purpose was to determine the polyphenol contents, antioxidant activities and major phenolic compound profiles of pomegranate co-products such as peel, press cake and precipitate after clarification which is obtained from industrial scale pasteurised pomegranate nectar processing and comparing with that of arils, and products such as concentrate and pasteurised nectar. Also, the other purpose was to compare the potential bioavailability of arils, co-products and final products after simulating in vitro GI digestion, for better understanding the real effects in human metabolism and the potential of co-products to be used in other applications.

\section{Materials and methods}

\section{Chemicals}

Gallic acid, Quercetin, Catechin, pepsin, pancreatin, bile salts, Quercetin-3- $\beta$-D-glucoside and Pelargonidin 3O-glucoside from Sigma-Aldrich (Steinheim, Germany); 6-hydroxy-2,5,7,8-tetramethylchroman-2-carboxylic acid (Trolox), Ferulic acid and Quercetin-3-O-galactoside from Fluka (Buchs, Switzerland); sodium bicarbonate from BDH Chemicals (Poole, UK); Cyanidin 3-O-glucoside, Pelargonidin 3,5-di-O-glucoside, Cyanidin 3,5-di-O-glucoside, Delphinidin 3-O-glucoside and Delphinidin 3,5-di-Oglucoside from Extrasynthese (Genay, France) were purchased for in vitro GI digestion and other analyses.

\section{Materials}

Pomegranate (cv. Hicaznar) samples were taken from industrial scale pasteurised pomegranate nectar (PN) manufacturing plant which processed pomegranate grown in Karaman region of Turkey. Duplicate samples were taken from two different productions. Processing steps were peeling, mashing of pomegranate arils to inactivate the enzymes $\left(80^{\circ} \mathrm{C}, 90 \mathrm{~s}\right)$, pressing, cooling $\left(4{ }^{\circ} \mathrm{C}, 25-30 \mathrm{~min}\right)$, pasteurisation $\left(90-95^{\circ} \mathrm{C}, 90 \mathrm{~s}\right)$, cooling (to $50-55^{\circ} \mathrm{C}$ ), enzyme application $(37.5 \mathrm{~mL}$ pectinolytic enzyme/ton juice), clarification ( $1.2 \mathrm{~kg}$ bentonite/ton juice and $780 \mathrm{~g}$ gelatin/ton juice at $50{ }^{\circ} \mathrm{C}$, $1 \mathrm{~h}$ ), ultrafiltration, evaporation (from $12.5^{\circ}$ to $65^{\circ} \mathrm{Brix}$ at $65-80{ }^{\circ} \mathrm{C}$ ), production of nectar by adding sucrose, citric acid and water and dilution to $12.5^{\circ} \mathrm{Brix}$, and pasteurisation of nectar $\left(95^{\circ} \mathrm{C}, 45 \mathrm{~s}\right)$, respectively. Samples were taken from pomegranate peel (PP), pomegranate arils (PA), press cake (PC), precipitate after clarification $(\mathrm{PAC})$, concentrate $(\mathrm{CON})$ and $\mathrm{PN}$. They were milled under liquid nitrogen using a grinder (IKA, Germany) and stored at $-80{ }^{\circ} \mathrm{C}$ until analysis.

\section{Moisture content analysis}

The analysis was performed as described in Association of Official Analytical Chemists (AOAC) 934.06 method (1997) to express the results in dry matter basis $(\mathrm{dmb})$.

\section{Extraction of phenolic compounds}

Extraction procedure was carried out according to Capanoglu et al. (2008). One gram of ground sample, except for PP $(0.5 \mathrm{~g})$, was extracted with $5 \mathrm{~mL}$ of $75 \%$ aqueous methanol including $0.1 \%(\mathrm{v} / \mathrm{v})$ formic acid in an ice-cooled ultrasonic bath (Ultrasonic CleanerVWR) for $15 \mathrm{~min}$. They were centrifuged (Hettich Universal 32, Tuttlingen, Germany) for $10 \mathrm{~min}$ at $2701 \mathrm{~g}$ under $4{ }^{\circ} \mathrm{C}$. It was repeated four times until $20 \mathrm{~mL}$ of extract was collected, and extracts were stored at $-20{ }^{\circ} \mathrm{C}$ until analysis.

\section{Spectrophotometric methods}

Total phenolic content (TPC), total flavonoid content (TFC), total tannin content (TTC), total anthocyanin content (TAC) and total antioxidant activity (TAA) analysis were carried out by spectrophotometric methods using UV-Visible spectrophotometer (Shimadzu, Japan).

Total phenolic content was determined using FolinCiocalteu method as described previously by Surek \& Nilufer-Erdil (2014). The results were expressed as $\mathrm{mg}$ Gallic acid equivalents (GAE) per $100 \mathrm{~g}$ of sample in $\mathrm{dmb}$.

Total flavonoid content was measured as described by Viuda-Martos et al. (2011). The results were expressed as mg Quercetin equivalents (QE) per $100 \mathrm{~g}$ of sample in dmb.

Total anthocyanin content and TTC were measured as described by Kar et al. (2011), and the results were 
expressed as mg Cyanidin 3-O-glucoside (Cyn 3-O-glu) and $\mathrm{mg}$ Catechin equivalents (CE) per $100 \mathrm{~g}$ of sample in $\mathrm{dmb}$, respectively.

Total antioxidant activity was determined by Cupric Reducing Antioxidant Capacity (CUPRAC), Ferric Reducing Ability of Plasma (FRAP), 2,2-diphenyl-1picrylhydrazyl (DPPH) and 2,2'-azinobis-3-ethylbenzothiazoline-6-sulphonic acid diammonium salt (ABTS) radical scavenging activity methods. The results were expressed as mg Trolox equivalents (TEAC) per $100 \mathrm{~g}$ of sample in dmb. CUPRAC, FRAP, ABTS and DPPH assays were performed according to Apak et al. (2006), Benzie \& Strain (1996), Miller \& Rice-Evans (1997) and Viuda-Martos et al. (2011), respectively.

\section{High-performance liquid chromatography (HPLC)- photodiode array (PDA) analysis of major phenolic compounds and anthocyanins}

Samples were filtered through a $0.45-\mu \mathrm{m}$ membrane filter and then injected into a Waters 2695 reversed phase HPLC system coupled with PDA Waters 2996 detector. Luna C18 column $(150 \times 4.60 \mathrm{~mm}$, pore size $100 \AA$, particle size $5 \mu \mathrm{m}$ from Phenomenex (Torrance, CA, USA)) was used as the stationary phase. The mobile phase consisted of solvent A, Milli-Q water with $0.1 \%$ (v/v) TFA, and solvent B, acetonitrile with $0.1 \%(\mathrm{v} / \mathrm{v})$ TFA. The HPLC running condition was based on the study of Surek \& Nilufer-Erdil (2014). Quantification was done using external standard curves.

\section{Simulated in vitro gastrointestinal digestion}

Gastrointestinal digestion was simulated to determine the potential bioavailability of polyphenols in samples. Bioavailability was assessed according to the protocol described by McDougall et al. (2005). Release of phytochemicals from pomegranate samples was analysed at different stages of digestion. These stages are the aliquots from gastric digestion (PG, postgastric) and GI digestion (IN and OUT). IN fraction (dialysed part) is the one that entered the serum while OUT fraction (undialysed fraction) is the part remaining in the colon. All fractions obtained were kept at $-20{ }^{\circ} \mathrm{C}$ until further analysis. Prior to analysis they were thawed and centrifuged at $23000 \mathrm{~g}$ for $20 \mathrm{~min}$. TPC, TAC and TAA analysis by DPPH besides phenolic and anthocyanin profiles were determined for each fraction. The results of extracts were assumed as $100 \%$, and $\%$ recovery was calculated for all fractions of all samples.

\section{Statistical analysis}

All of the results were reported as mean value \pm standard deviation. Data from duplicate independent samples of two treatments were analysed statistically using one-way analysis of variance (ANOVA). When significant differences were observed $(P<0.05)$, means were subjected to Duncan's new multiple range test. Analysis was conducted using Statistical Package for the Social Sciences software (version 16.0, SPSS Inc., Chicago, IL, USA).

\section{Results and discussion}

Total phenolic, flavonoid, anthocyanin and tannin contents

Results obtained for TPC, TAC, TFC and TTC analysis are given in Table 1. PP being the major waste of processing showed significantly highest TPC, TFC and TTC values; however, for TAC, PA was higher than all $(P<0.05)$. Nectar production step which includes addition of water and sugar resulted in significant losses in all parameters for PN, being the end product when compared to co-products $(P<0.05)$. Viuda-Martos et al. (2011) determined TPC, TFC and TTC of bagasse from pomegranate arils and whole fruit as $4.6 \pm 0.7$ and $10.0 \pm 1.2 \mathrm{mg} \mathrm{GAE} / \mathrm{g}$ fresh weight (FW); $5.7 \pm 0.3$ and $7.2 \pm 0.5 \mathrm{mg}$ Rutin equivalents $/ \mathrm{g}$ $\mathrm{FW}$; and $6.7 \pm 0.3$ and $8.2 \pm 0.1 \mathrm{mg} \mathrm{CE} / \mathrm{g} \mathrm{FW}$, respectively. In this study, TPC, TFC and TTC of PC were calculated as $14.5 \pm 0.7 \mathrm{mg} \mathrm{GAE} / \mathrm{g} \mathrm{FW}$, $15.1 \pm 0.9 \mathrm{mg}$ QE $/ \mathrm{g} F W$ and $1.8 \pm 0.2 \mathrm{mg} \mathrm{CE} / 100 \mathrm{~g}$ $\mathrm{FW}$, respectively, which were different than their study, due to difference in pomegranate variety and production procedure. Ardekani et al. (2011), Li et al. (2006) and Nasr et al. (1996) measured TPC of PP extract as $98.2 \pm 4.8-226.6 \pm 18.9 \mathrm{mg}$ GAE $/ \mathrm{g} \mathrm{DW}$, $249 \pm 17.2 \mathrm{mg}$ Tannic acid $/ \mathrm{g}$ FW and $216 \pm 7.3 \mathrm{mg}$ GAE/g DW. Gullon et al. (2015) reported TPC of PP flour as $19.30 \mathrm{mg}$ GAE/g DW. In this study, TPC of PP was found as $180.3 \pm 15.7 \mathrm{mg}$ GAE $/ \mathrm{g}$ DW $(55.3 \pm 4.8 \mathrm{mg}$ GAE $/ \mathrm{g} \mathrm{FW})$, which were consistent with Ardekani et al. (2011), much higher than Gullon et al. (2015) and lower than others. TPC can be different according to several factors such as the cultivar, growing region, climate, maturity, cultivation practice, processing and storage conditions, analysis and extraction methodology used or the solvents employed (Viuda-Martos et al., 2011; Gullon et al., 2015). Ardekani et al. (2011) reported that TFC for PP in the range of $18.6 \pm 0.5$ to $36.4 \pm 1.3 \mathrm{mg} \mathrm{CE} / \mathrm{g}$ DW and $\mathrm{Li}$ et al. (2006) found $59.1 \pm 4.8 \mathrm{mg}$ Rutin/g FW. TFC of PP flour was obtained as $12.27 \mathrm{mg}$ Rutin/g DW by Gullon et al. (2015). In this study, TFC of PP was closer to Li et al. (2006), but higher than Ardekani et al. (2011) and Gullon et al. (2015). Preparing calibration curves with different standards is also one of the major reasons for the different findings (Surek $\&$ Nilufer-Erdil, 2014). There was no significant difference between TAC values of PP and PC $(P>0.05)$. 
Elfalleh et al. (2011) reported TAC of Tunisian PP as $73.9 \pm 8.2 \mathrm{mg}$ Cyn 3-O-glu/g DW which was higher than the value in this study $(51.8 \pm 6.4 \mathrm{mg} / 100 \mathrm{~g}$ DW). In particular, TAC in PAC was found to be very high; on the other hand, significant loss in the final product was evident $(P<0.05)$. A previous study by Turfan et al. (2012) reported that the reason of the loss of TAC after clarification of pomegranate juice was the interaction between anthocyanins and gelatintannin flocks. Mousavinejad et al. (2009) quantified TTC of pomegranate juices produced in laboratory as $0.01-0.03 \mathrm{~g} / 100 \mathrm{~g} \mathrm{FW}$, which was lower than this study maybe because of using different raw material and producing with different techniques. This study showed that both after pressing and clarification coproducts were found to possess similar amounts of TPC, TFC and TTC with CON $(P>0.05)$ and significant higher levels when compared to PN $(P<0.05)$. On the other hand, they were significantly lower than PP and PA $(P<0.05)$.

\section{Total antioxidant activity}

Total antioxidant activity analysis by DPPH, CUPRAC, FRAP and ABTS methods is shown in Table 1. In all methods used for the determination of TAA, PP showed higher antioxidant activity than other samples, statistically $(P<0.05)$. This may be explaining because PP had greater concentration of phenols, flavonoids and tannins than others. Therefore, discarding the peel, which is rich in polyphenols, caused important loss in TAA $(P<0.05)$. TAA was significantly lower in the PA, which is used as the raw material, with respect to the PP which is discarded $(P<0.05)$. On the other hand, TAA values for $\mathrm{CON}$, $\mathrm{PC}$ and PAC were found to be 2.5-fold to 4.5-fold higher than the TAA of PN which is consumed as the product $(P<0.05)$. According to CUPRAC method, TAA of PC, CON and PAC were lower than PP and PA $(P<0.05)$. By DPPH and FRAP method, TAA was not found to be different for CON, PC and PAC $(P>0.05)$. By ABTS method differently, TAA of CON was found to be significantly different than PC and PAC $(P<0.05)$. Capanoglu et al. (2008) reported that TAA of seed and skin of tomato by DPPH was different from CUPRAC but similar to FRAP methods. In the current study, also DPPH results were generally consistent with FRAP. According to FRAP method, PA, CON, PC and PAC showed similar TAA values $(P>0.05)$. When overall evaluation for TAA values was done, it was obvious that CUPRAC method had given the highest responses for TAA followed by DPPH or ABTS. FRAP method generally had the lowest TAA values for those samples. Four assays were applied to assess TAA of pomegranate co-products; however, the values obtained were

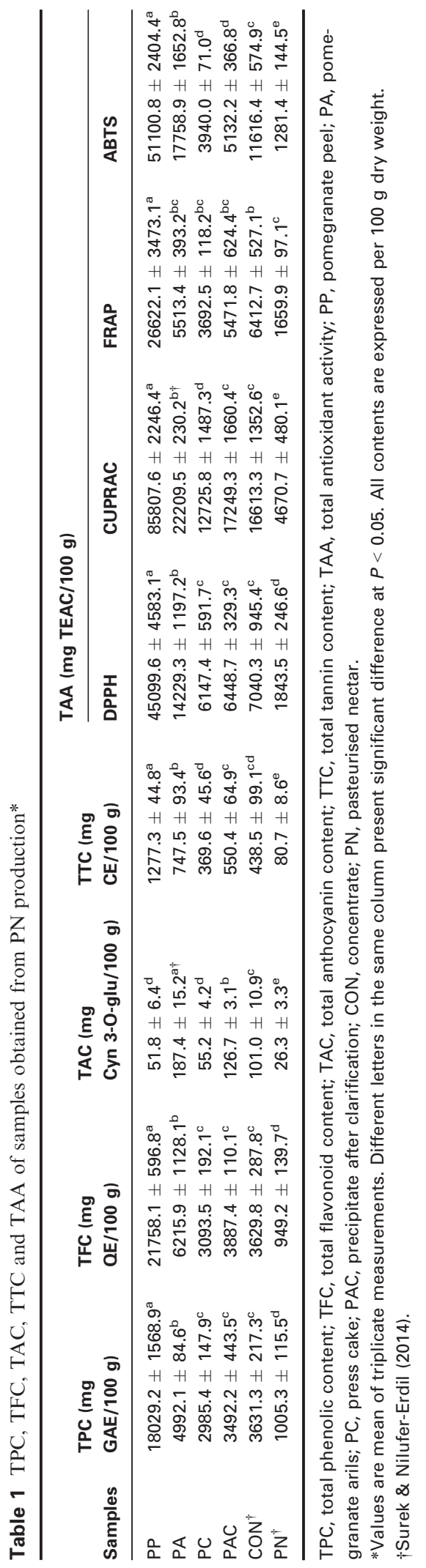

C 2015 Institute of Food Science and Technology 
different. It is very difficult to assess TAA of a product on the basis of a single method because the antioxidant mechanism is quite complex and many factors such as solvent, temperature, chemical structure of phenolics and $\mathrm{pH}$ of the medium play a role in these mechanisms (Huang et al., 2005; Viuda-Martos et al., 2011). The DPPH assay is based on the reduction of the purple DPPH radical to 1,1-diphenyl-2-picryl hydrazine, which is applicable to hydrophobic systems. ABTS assay has the similar principle with that of DPPH, whereas it is based on the production of stable a blue/green $\mathrm{ABTS}^{+}$, which is applicable to both hydrophilic and lipophilic systems by reacting ABTS (pH 4.5) (Floegel et al., 2011; Wootton-Beard et al., 2011). On the other hand, no free radicals are involved in the FRAP and CUPRAC assays, but the reduction of $\mathrm{Fe}^{3+}$ to $\mathrm{Fe}^{2+}$ at $\mathrm{pH} 3.6$ and the reduction of $\mathrm{Cu}^{2+}$ to $\mathrm{Cu}^{+}$at $\mathrm{pH} 7.0$ are observed, respectively (Apak et al., 2006; Floegel et al., 2011). The CUPRAC assay was claimed as more useful than FRAP by Apak et al. (2006) due to having end point mechanism. On the other hand, FRAP assay may not be complete even many hours, and thus, a single end point of the reaction cannot be determined (Prior et al., 2005). The ABTS assay is an end point assay; however, different antioxidant compounds donate one or two electrons to reduce the radical cation (Wootton-Beard et al., 2011). Therefore, the measurement of TAA cannot be evaluated sufficiently by a single method, and a mixture of assays should be used.

\section{Major phenolic compounds}

Results obtained for major phenolic compounds analysis by HPLC-PDA are shown in Table 2. Major compounds were identified as Gallic acid, Catechin, Ferulic acid, Quercetin-3-O-galactoside (Q-3-g), Quercetin-3- $\beta$-D-glucoside (Q-3- $\beta \mathrm{Dg})$, Delphinidin 3,5-di-Oglucoside (Del 3,5-dOg), Cyanidin 3-O-glucoside (Cyn 3-O-glu), Pelargonidin 3,5-di-O-glucoside (Pel 3,5-dOg) and Pelargonidin 3-O-glucoside (Pel 3-O-glu). Gallic acid, Ferulic acid, Q-3- $\beta$ Dg and Cyn 3-O-glu were identified in all of the samples. Catechin was not detected in CON and PAC while PC showed similar levels to PA $(P>0.05)$ and greater than PN $(P<0.05)$. Gallic acid was identified in all samples being at the highest level in PP and followed by PA and CON. Gallic acid concentration of PC was similar to PAC and CON $(P>0.05)$. Elfalleh et al. (2011) detected $123.79 \pm 9.56 \mathrm{mg} / 100 \mathrm{~g}$ DW Gallic acid in Tunisian PP, which was lower than this study. Differences in phenolic acids concentration can be dependent on pomegranate variety and growth conditions. PP and PA showed the highest ferulic acid concentrations; however, PN had the lowest. Similar to gallic acid, no difference was observed between ferulic acid levels of
CON and PC $(P>0.05)$. Q-3-g was found in PP and in mostly lesser amounts in PA, PC and CON; however, in PN and PAC, it was not identified. Q-3- $\beta$ Dg was at the highest level in PP and followed by PA. On the other hand, no significant difference was found for its content in PC and PAC $(P>0.05)$ while PN had lowest Q-3- $\beta$ Dg levels as the product.

For anthocyanins; PA and CON had significantly higher values than PP $(P<0.05)$. PN had also significantly lower values than PA and CON $(P<0.05)$. PAC, CON and PC had similar values for major anthocyanins such as Cyn 3-O-glu and Pel 3,5-dOg. PA showed the highest and similar Del 3,5-dOg concentration with CON $(P>0.05)$. PN and PC showed the same Del 3,5-dOg concentrations $(P>0.05)$. Pel $3,5-\mathrm{dOg}$ was only found in PA, CON, PAC and PC. Significantly important levels were retained in co-products $(P<0.05)$. Ozkal \& Dinc $(1993)$ observed that Pel 3,5-dOg and Pel 3-O-glu were present in high values in PP and in low amounts in PA. They also claimed that both Cyanidin 3,5-di-O-glucoside (Cyn 3,5-dOg) and Cyn 3-O-glu were detected in PP, but Del 3,5-dOg and Del 3-O-glu which were major anthocyanins of pomegranate juice could not be detected in PP. In this study, Pel 3,5-dOg could not be found in PP, while Pel 3-O-glu was only detected in PP. Cyn 3,5-dOg could not be found in PA and PP; however, Cyn 3-O-glu was detected for each and PA was higher than others. Delphinidin 3-O-glucoside (Del 3-O-glu) and Del 3,5-dOg could not be detected in PP as reported by Ozkal \& Dinc (1993). There are also some studies in which more anthocyanins of pomegranate juice were detected. Gil et al. (2000) and Turfan et al. (2011) detected Del 3,5-dOg, Cyn 3,5-dOg, Cyn 3-Oglu, Pel 3-O-glu and Del 3-O-glu in juices. Anthocyanin concentration can be degraded or reduced during processing and storage and depends on growth conditions and variety as well (Ozkal \& Dinc, 1993).

\section{Bioaccessibility evaluation by in vitro gastrointestinal digestion}

Evaluations of bioaccessibility for the samples (PG, IN and OUT fractions) after in vitro GI digestion are given in Table 3. PP and PN had the highest and the lowest TPC values for all fractions, respectively. No significant difference was found between $\mathrm{CON}$ and PC according to $\mathrm{PG}$ fraction which is representing the part leaving the stomach $(P>0.05)$. For the IN fraction, TPC of PA and PAC were statistically similar $(P>0.05)$. There was no significant difference between TPC of OUT fractions of PA and PAC, and CON and PC $(P>0.05)$. Most phenolics were discarded as by products or wastes during pomegranate processing. However, very low bioaccessibility was obtained for PN. \% recovery values in PG fraction were very high 
Table 2 Major phenolic compound analysis by HPLC-PDA of samples obtained from PN production (mg/100 g DW)*

\begin{tabular}{|c|c|c|c|c|c|}
\hline Samples & Gallic Acid & Catechin & Ferulic Acid & Q-3-g & 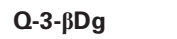 \\
\hline PP & $213.8 \pm 57.4^{\mathrm{a}}$ & $6954.6 \pm 865.0^{\mathrm{a}}$ & $65.0 \pm 7.0^{\mathrm{a}}$ & $117.1 \pm 12.4^{\mathrm{a}}$ & $400.5 \pm 5.9^{a}$ \\
\hline PA & $156.8 \pm 6.7^{\mathrm{b}}$ & $506.5 \pm 96.5^{\mathrm{b}}$ & $47.6 \pm 4.2^{\mathrm{b}}$ & $9.1 \pm 0.0^{\mathrm{b}}$ & $326.5 \pm 26.7^{\mathrm{b} \dagger}$ \\
\hline PC & $71.2 \pm 0.1^{\mathrm{cd}}$ & $359.9 \pm 52.0^{b}$ & $28.8 \pm 1.7^{c}$ & $13.7 \pm 0.9^{b}$ & $108.4 \pm 14.0^{c}$ \\
\hline PAC & $61.9 \pm 5.9^{d}$ & ND & $20.6 \pm 1.9^{d}$ & ND & $88.0 \pm 8.2^{c}$ \\
\hline $\mathrm{CON}^{+}$ & $100.4 \pm 10.4^{c}$ & ND & $31.8 \pm 4.1^{c}$ & $4.9 \pm 0.1^{b}$ & $61.0 \pm 0.0^{\mathrm{d}}$ \\
\hline $\mathrm{PN}^{\dagger}$ & $26.1 \pm 2.3^{\mathrm{e}}$ & $10.5 \pm 0.0^{b}$ & $5.4 \pm 0.5^{\mathrm{e}}$ & ND & $21.0 \pm 1.6^{\mathrm{e}}$ \\
\hline
\end{tabular}

\begin{tabular}{lllll}
\hline & \multicolumn{2}{c}{ Anthocyanins } & & \\
\cline { 2 - 5 } Samples & Cyn 3-O-glu & Del 3,5-dOg & Pel 3,5-dOg & PD \\
PP & $15.2 \pm 0.3^{\mathrm{c}}$ & $\mathrm{ND}$ & $103.4 \pm 28.6^{\mathrm{a}}$ & $12.4 \pm 0.8$ \\
$\mathrm{PA}$ & $42.6 \pm 4.9^{\mathrm{a} \dagger}$ & $41.0 \pm 4.1^{\mathrm{a} \dagger}$ & $14.6 \pm 1.4^{\mathrm{b}}$ & $\mathrm{ND}$ \\
$\mathrm{PC}$ & $24.9 \pm 0.1^{\mathrm{b}}$ & $10.9 \pm 1.5^{\mathrm{c}}$ & $34.6 \pm 5.3^{\mathrm{b}}$ & $\mathrm{ND}$ \\
$\mathrm{PAC}$ & $20.5 \pm 1.0^{\mathrm{bc}}$ & $23.0 \pm 5.2^{\mathrm{b}}$ & $38.3 \pm 3.7^{\mathrm{b}}$ & $\mathrm{ND}$ \\
$\mathrm{CON}^{\dagger}$ & $21.0 \pm 0.2^{\mathrm{b}}$ & $35.6 \pm 0.0^{\mathrm{a}}$ & $\mathrm{ND}$ & $\mathrm{ND}$ \\
$\mathrm{PN}^{\dagger}$ & $5.5 \pm 0.7^{\mathrm{d}}$ & $13.0 \pm 2.1^{\mathrm{c}}$ & & $\mathrm{ND}$ \\
\hline
\end{tabular}

PP, pomegranate peel; PA, pomegranate arils; PC, press cake; PAC, precipitate after clarification; CON, concentrate; PN, pasteurised nectar; Q-3-g, Quercetin-3-O-galactoside; Q-3- $\beta \mathrm{Dg}$, Quercetin-3- $\beta$-D-glucoside; Cyn 3-O-Glu, Cyanidin 3-O-glucoside; Del 3,5-dOg, Delphinidin 3,5-di-O-glucoside; Pel 3,5-dOg, Pelargonidin 3,5-di-O-glucoside; Pel-3-O-glu, Pelargonidin 3-O-glucoside; ND, not detected.

*Values are mean of triplicate measurements. Different letters in the same column present significant difference at $P<0.05$.

$\dagger$ Surek \& Nilufer-Erdil (2014).

Table 3 The concentrations and per cent recoveries of TPC, TAC and TAA by DPPH of samples after in vitro GI digestion*

\begin{tabular}{|c|c|c|c|c|c|c|c|c|c|c|c|c|}
\hline \multirow[b]{2}{*}{ Samples } & \multicolumn{6}{|c|}{ TPC (mg GAE/100 g DW) } & \multicolumn{6}{|c|}{ TAC (mg Cyn 3-O-glu/100 g DW) } \\
\hline & PG & $\%$ & IN & $\%$ & OUT & $\%$ & PG & $\%$ & IN & $\%$ & OUT & $\%$ \\
\hline PP & $14511.5 \pm 1271.3^{\mathrm{a}}$ & 80.5 & $2295.6 \pm 54.5^{\mathrm{a}}$ & 12.7 & $20242.3 \pm 1068.2^{\mathrm{a}}$ & 112.3 & $40.7 \pm 4.3^{d}$ & 78.6 & $0.3 \pm 0.0^{\mathrm{b}}$ & 0.6 & $8.8 \pm 1.0^{\mathrm{d}}$ & 17.0 \\
\hline PA & $6444.1 \pm 432.0^{b}$ & 129.1 & $1113.1 \pm 52.2^{\mathrm{c}}$ & 22.3 & $6540.4 \pm 499.3^{\mathrm{b}}$ & 131.0 & $118.2 \pm 11.4^{\mathrm{a}}$ & 63.1 & $2.1 \pm 0.1^{\mathrm{a}}$ & 1.1 & $14.2 \pm 1.9^{c}$ & 7.6 \\
\hline PC & $2909.3 \pm 108.3^{d}$ & 97.5 & $512.0 \pm 63.2^{\mathrm{d}}$ & 17.2 & $4480.7 \pm 650.1^{c}$ & 150.1 & $22.3 \pm 5.7^{\mathrm{e}}$ & 40.4 & $0.6 \pm 0.1^{\mathrm{b}}$ & 1.1 & - & - \\
\hline PAC & $4575.1 \pm 198.9^{c}$ & 131.0 & $1004.6 \pm 86.8^{\mathrm{c}}$ & 28.8 & $7163.2 \pm 345.4^{\mathrm{b}}$ & 205.1 & $67.8 \pm 6.1^{\mathrm{c}}$ & 53.5 & $2.0 \pm 0.7^{\mathrm{a}}$ & 1.6 & $32.9 \pm 0.0^{\mathrm{a}}$ & 26.0 \\
\hline CON & $3569.3 \pm 67.1^{\mathrm{cd}}$ & 98.3 & $1559.7 \pm 192.3^{\mathrm{b}}$ & 43.0 & $3719.0 \pm 250.7^{c}$ & 102.4 & $87.0 \pm 3.6^{b}$ & 86.1 & $2.1 \pm 0.2^{\mathrm{a}}$ & 2.1 & $24.9 \pm 2.9^{b}$ & 24.7 \\
\hline PN & $968.3 \pm 169.5^{\mathrm{e}}$ & 96.3 & $196.6 \pm 11.4^{\mathrm{e}}$ & 19.6 & $54.8 \pm 9.5^{d}$ & 5.5 & $2.4 \pm 0.4^{f}$ & 9.1 & $0.4 \pm 0.1^{\mathrm{b}}$ & 1.5 & $2.7 \pm 0.9^{\mathrm{e}}$ & 10.3 \\
\hline
\end{tabular}

\begin{tabular}{|c|c|c|c|c|c|c|}
\hline \multirow[b]{2}{*}{ Samples } & \multicolumn{6}{|c|}{ TAA by DPPH (mg TEAC/100 g DW) } \\
\hline & PG & $\%$ & IN & $\%$ & OUT & $\%$ \\
\hline PP & $2332.3 \pm 168.4^{\mathrm{a}}$ & 5.2 & $22.9 \pm 1.2^{\mathrm{e}}$ & 0.1 & $2024.6 \pm 293.7^{\mathrm{a}}$ & 4.5 \\
\hline PA & $2184.7 \pm 116.7^{\mathrm{a}}$ & 15.4 & $126.3 \pm 0.5^{\mathrm{a}}$ & 0.9 & $1329.5 \pm 245.7^{\mathrm{b}}$ & 9.3 \\
\hline PC & $1158.7 \pm 8.0^{\mathrm{b}}$ & 18.8 & $45.1 \pm 4.0^{c}$ & 0.7 & $611.5 \pm 4.7^{c}$ & 9.9 \\
\hline PAC & $2337.8 \pm 292.4^{\mathrm{a}}$ & 36.3 & $114.2 \pm 7.9^{\mathrm{b}}$ & 1.8 & $2011.9 \pm 31.0^{\mathrm{a}}$ & 31.2 \\
\hline CON & $1012.6 \pm 70.3^{\mathrm{b}}$ & 14.4 & $33.8 \pm 2.4^{\mathrm{d}}$ & 0.5 & $661.3 \pm 60.2^{\mathrm{c}}$ & 9.4 \\
\hline PN & $2013.7 \pm 117.1^{\mathrm{a}}$ & 109.2 & $44.4 \pm 3.3^{c}$ & 2.4 & $350.4 \pm 34.0^{\mathrm{c}}$ & 19.0 \\
\hline
\end{tabular}

TPC, total phenolic content; TAC, total anthocyanin content; TAA, total antioxidant activity; PP, pomegranate peel; PA, pomegranate arils; PC, press cake; PAC, precipitate after clarification; CON, concentrate; PN, pasteurised nectar; PG, the fraction leaving the stomach; OUT, the fraction going into colon; IN, the fraction absorbed in serum; \%, recovery values were calculated by assuming the extract values as $100 \%$.

*Values are mean of triplicate measurements. Different letters in the same column present significant difference at $P<0.05$.

for PA and PAC. Although PP showed the greatest level of TPC for the extract, and even IN, PG and OUT fractions, it had the lowest \% recovery in IN fraction $(12.7 \%)$ when compared to the extract. For OUT fraction, recovery values were found to be so low $(5.5 \%)$ for the final product although all other samples had very high recovery values. For TAC values, PA had the highest values in PG fraction whereas for other fractions, especially, for IN no significant difference was obtained between PA, PAC and CON 
$(P>0.05)$; PP, $\mathrm{PC}$ and $\mathrm{PN}$ as the anthocyanins passed into serum at very low levels $(P>0.05)$. PN had the lowest values in PG and OUT fractions. IN values were lower than the OUT for all samples. When \% recovery for TAC is evaluated for IN fraction, all samples had low recoveries $(0.6-2.1 \%)$, whereas for PG fraction, recoveries were determined in the range of (9.1-86.1\%) CON being the highest, and thus, potential anthocyanin bioavailability was at very low levels. According to TPC results by Perez-Vicente et al. (2002), pepsin digestion did not change the values; however, $29 \%$ of TPC were detected in IN after pancreatin-bile salt digestion. Similar results were found for PA and PAC in this study; and for arils, $129 \%$ of TPC were found in PG. There was an increase in TPC compared to initial values for some samples after gastric digestion. This increase can be due to acidic hydrolysis of the phenolic glycosides to their aglycons during gastric digestion Perez-Vicente et al. (2002) reported that $\%$ recovery values for anthocyanin bioaccessibility in IN and in OUT fractions were 2.4 and $15.3 \%$, respectively. These values were also in agreement with the ones detected in this study. McDougall et al. (2005) reported that only $5.3 \%$ of TAC and $10.3 \%$ of TPC of raspberry were recovered after digestion. Fazzari et al. (2008) determined increase in TPC at PG fraction for frozen sweet cherries; and in IN fraction, TPC \% recovery values were about $26-30 \%$. Some of pomegranate samples in this study showed higher TPC \% recovery and lower TAC $\%$ recovery than McDougall et al. (2005) and Fazzari et al. (2008). The reason for high loss of anthocyanins is not completely known, but there can be some factors such as chemical conversions of anthocyanins (Perez-Vicente et al., 2002). It could be due to release of phenolic compounds from matrix after pepsin digestion. Anthocyanin stability is dependent on $\mathrm{pH}$ conditions; at low $\mathrm{pH}$, anthocyanins occur in the red form of flavylium cation. On the other hand, comparisons of in vitro studies are not easy because of the differences in material and in vitro digestion applied (Fazzari et al., 2008). The bioavailability of pomegranate polyphenols is affected by many factors such as production conditions of juice, analysis methods and variety of the fruit used (Basu \& Penugonda, 2009).

Total antioxidant activity obtained by DPPH method after GI digestion showed that for PG fraction PP, PA, PAC and PN had similar TAA values $(P>0.05)$. PA showed also higher TAA values in IN fraction $(P<0.05)$. For $\mathrm{IN}$ fraction, $\mathrm{PC}$ and $\mathrm{PN}$ showed similar TAA values $(P>0.05)$. PP had the lowest TAA value and \% recovery of IN fraction. For OUT fraction, PP and PAC and CON, PC and PN were not significantly different $(P>0.05)$. IN \% recovery values for all samples were very low $(0.1-$ $2.4 \%$ ). PG \% recovery of PN, PAC and PC was high, respectively. Although $\mathrm{CON}$ showed higher total phenolic bioaccessibility, it had low TAA.

Results for major phenolic compound analysis by HPLC-PDA of samples after in vitro digestion are given in Table 4. Anthocyanins were not be detected for any IN and OUT of samples, so phenolic bioaccessibility was detected higher than anthocyanin bioaccessibility. PP had the highest values of PG, IN and OUT of Gallic acid, Catechin, Ferulic acid, Q-3-g and Q-3$\beta D g$ due to also showing the highest values for extracts. IN values were lower than OUT for all samples. As reported by Perez-Vicente et al. (2002), the reason of the significant loss of anthocyanins is not completely known, but they can be metabolised to noncoloured forms, oxidised or degraded into other chemicals which cannot be detected under these conditions. It is also important that no aglycons occur after pancreatin-bile (intestinal) digestion (Perez-Vicente et al., 2002). Gallic acid was only detected for IN of $\mathrm{PP}$ and PA. Catechin values in PG of PP, PA, PC, PAC, CON and PN were measured as $1405.3 \pm 291.9$, $668.5 \pm 13.5, \quad 236.1 \pm 16.4, \quad 297.9 \pm 16.2, \quad 379.1 \pm$ 16.2 and $121.3 \pm 11.4 \mathrm{mg} / 100 \mathrm{~g} \mathrm{DW}$, respectively. There was no Catechin in any IN and PP showed the only OUT value as $21390.2 \pm 4500.2 \mathrm{mg} / 100 \mathrm{~g} \mathrm{DW}$.

Pel 3,5-dOg for PN and Pel 3-O-glu for PA, CON, PAC and PC were detected for PG samples, although they were not found for extracts. Generally, there was an increase in PG for phenolic compounds and anthocyanins, however, excessive decrease in IN and OUT for anthocyanins. This was probably due to enhancer effect of enzymes such as pepsin and acidic $\mathrm{pH}$ of the medium in gastric conditions, and effect of pancreatinbile mixtures and alkaline $\mathrm{pH}$ of the medium in intestinal conditions during digestion (Bermudez-Soto et al., 2007). Gastric conditions are effective on the release of some bound anthocyanins by acidic hydrolysis.

\section{Conclusion}

In general, phenolic content, antioxidant activities and potential bioaccessibilities of co-products obtained from industrial PN processing were determined in this study. This is the first study to investigate antioxidant potential and bioaccessibilities of co-products such as $\mathrm{PC}$ and PAC in comparison with raw materials and final products. PA and PAC were good sources for anthocyanins. It is evident that the results obtained with our in vitro GI method has shown that co-products such as PP, PC and PAC have significant levels of phenolics and antioxidant activity as much as or even higher than products. They can be used as a functional ingredient in dietary supplements or food formulations. Although in vitro digestion methods are preferred because of their advantages such as low cost, rapidity and simplicity for investigating potential 


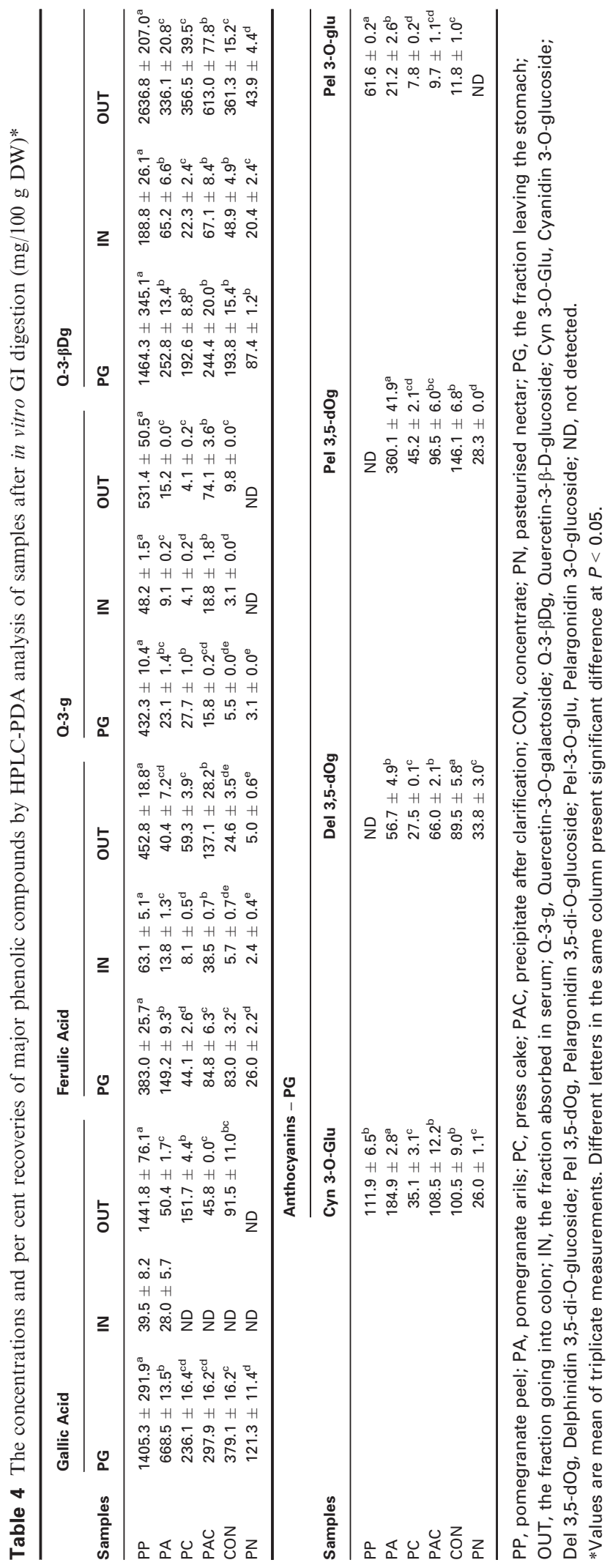

bioaccessibility, they cannot exactly predict the human in vivo conditions. Therefore, further in vivo studies should also be performed to support the findings of in vitro studies.

\section{Acknowledgment}

This study was supported by EU 7th Frame ATHENA Project. The authors thank AROMA Fruit Juices and Food Industry Inc. Karaman Facility for giving the chance to take samples from the processing lines and carry out such a comprehensive project. The authors are grateful to Dr. Esra Capanoglu Guven and Prof. Dr. Dilek Boyacioglu for their concern, support and help in obtaining the samples in this study.

\section{References}

Adsule, R.N. \& Patil, N.B. (1995). Pomegranate. In: Handbook of Fruit Science and Technology. (edited by D.K. Salunkhe \& S.S. Kadam) Pp. 455-462 New York: Marcel Dekker.

Alighourchi, H., Bargezar, M. \& Abbasi, S. (2008). Anthocyanins characterization of 15 Iranian pomegranate (Punica granatum L.) varieties and their variation after cold storage and pasteurization. European Food Research and Technology, 227, 881-887.

Alper, N., Bahceci, K.S. \& Acar, J. (2005). Influence of processing and pasteurization of color values and total phenolic compounds of pomegranate juice. Journal of Food Processing and Preservation, 29, 357-368.

AOAC. (1997). Method 934.06. Official Methods of Analysis of the Association of Official Analytical Chemists, 16th edn. Gaithersburg, MD: AOAC International.

Apak, R., Guclu, K., Ozyurek, M. \& Karademir, S.E. (2006). The cupric ion reducing antioxidant capacity (CUPRAC) and polyphenolic content of some herbal teas. International Journal of Food Sciences and Nutrition, 7, 292-304.

Ardekani, M.R.S., Hajimahmoodi, M., Oveisi, M.R. et al. (2011). Comparative antioxidant activity and total flavonoid content of Persian pomegranate (Punica granatum L.) cultivars. Iranian Journal of Pharmaceutical Research, 10, 519-524.

Basu, A. \& Penugonda, K. (2009). Pomegranate juice: a hearthealthy fruit juice. Nutrition Reviews, 67, 49-56.

Benzie, I.F.F. \& Strain, J.J. (1996). The ferric reducing ability of plasma (FRAP) as measure of "antioxidant power": the FRAP assay. Analytical Biochemistry, 239, 70-76.

Bermudez-Soto, M.J., Tomas-Barberan, F.A. \& Garcia-Conesa, M.T. (2007). Stability of polyphenols in chokeberry (Aronia melanocarpa) subjected to in vitro gastric and pancreatic digestion. Food Chemistry, 102, 865-874.

Capanoglu, E., Beekwilder, J., Boyacioglu, D., Hall, R. \& De Vos, R. (2008). Changes in antioxidant and metabolite profiles during production of tomato paste. Journal of Agricultural and Food Chemistry, 56, 964-973.

Elfalleh, W., Tlili, N., Nasri, N. et al. (2011). Antioxidant capacities of phenolic compounds and tocopherols from Tunisian pomegranate (Punica granatum) fruits. Journal of Food Science, 76, 707713.

Fazzari, M., Fukumoto, L., Mazza, G., Livrea, M.A., Tesoriere, L. \& Marco, L.D. (2008). In vitro bioavailability of phenolic compounds from five cultivars of frozen sweet cherries (Prunus avium L.). Journal of Agricultural and Food Chemistry, 56, 35613568.

Floegel, A., Kim, D.O., Chung, S.J., Koo, S.I. \& Chun, O.K. (2011). Comparison of ABTS/DPPH assays to measure antioxidant 
capacity in popular antioxidant-rich US foods. Journal of Food Composition and Analysis, 24, 1043-1048.

Gil, M.I., Barberan, F.A.T., Pierce, B.H., Holcroft, D.M. \& Kader, A.A. (2000). Antioxidant activity of pomegranate juice and its relationship with phenolic composition and processing. Journal of Agricultural and Food Chemistry, 48, 4581-4589.

Gullon, B., Pintado, E., Perez-Alvarez, J.A. \& Viuda-Martos, M. (2015). Assessment of polyphenolic profile and antibacterial activity of pomegranate peel (Punica granatum) flour obtained from coproduct of juice extraction. Food Control, 59, 94-98.

Huang, D., Ou, B. \& Prior, R.L. (2005). The chemistry behind antioxidant capacity assays. Journal of Agricultural and Food Chemistry, 53, 1841-1856.

Kar, C.E., Ferchichi, A., Attia, F. \& Bouajila, J. (2011). Pomegranate (Punica granatum) juices: chemical composition, micronutrient cations and antioxidant capacity. Journal of Food Science, 76, 795800.

Lee, J. \& Wrolstad, R.E. (2004). Extraction of anthocyanins and polyphenolics from blueberry processing waste. Journal of Food Science, 69, C564-C573.

Li, Y., Guo, C., Yang, J., Wei, J., Xu, J. \& Cheng, S. (2006). Evaluation of antioxidant properties of pomegranate peel extract in comparison with pomegranate pulp extract. Food Chemistry, 96, 254 260.

McDougall, G.J., Dobson, P., Smith, P., Blake, A. \& Stewart, D. (2005). Assessing potential bioavailability of raspberry anthocyanins using an in vitro digestion system. Journal of Agricultural and Food Chemistry, 53, 5896-5904.

Miller, N.J. \& Rice-Evans, C.A. (1997). Factors influencing the antioxidant activity determined by the $\mathrm{ABTS}^{+}$radical cation assay. Free Radical Research, 26, 195-199.

Mousavinejad, G., Emam-Djomeh, Z., Rezaei, K. \& Khodaparast, M.H.H. (2009). Identification and quantification of phenolic compounds and their effects on antioxidant activity in pomegranate juices of eight Iranian cultivars. Food Chemistry, 115, 1274 1278.

Nasr, C.B., Ayed, N. \& Metche, M. (1996). Quantitative determination of the polyphenolic content of pomegranate peel. Zeitschrift für Lebensmittel-Untersuchung und Forschung, 203, 374-378.

Ozkal, N. \& Dinc, S. (1993). Chemical composition and biological activities of Punica granatum L. (Pomegranate). Journal of Faculty of Pharmacy of Ankara, 22, 38-50.

Perez-Vicente, A., Gil-Izquierdo, A. \& Garcia-Viguera, C. (2002). In vitro gastrointestinal digestion study of pomegranate juice phenolic compounds, anthocyanins and vitamin C. Journal of Agricultural and Food Chemistry, 50, 2308-2312.

Prior, R.L., Wu, X. \& Schaich, K. (2005). Standardized methods for the determination of antioxidant capacity and phenolics in foods and dietary supplements. Journal of Agricultural and Food Chemistry, 53, 4290-4302.
Rinaldi, M., Caligiani, A., Borgese, R., Palla, G., Barbanti, D. \& Massini, R. (2013). The effect of fruit processing and enzymatic treatments on pomegranate juice composition, antioxidant activity and polyphenols content. LWT-Food Science and Technology, 53, $355-359$.

Selcuk, N. \& Erkan, M. (2015). Changes in phenolic compounds and antioxidant activity of sour-sweet pomegranates cv. 'Hicaznar' during long-term storage under modified atmosphere packaging. Postharvest Biology and Technology, 109, 30-39.

Sengul, H., Surek, E. \& Nilufer-Erdil, D. (2014). Investigating the effects of food matrix and food components on bioaccessibility of pomegranate (Punica granatum) phenolics and anthocyanins using an in-vitro gastrointestinal digestion model. Food Research International, 62, 1069-1079.

Surek, E. \& Nilufer-Erdil, D. (2014). Changes in phenolics and antioxidant activity at each step of processing from pomegranate into nectar. International Journal of Food Sciences and Nutrition, 65, 194-202.

Suzme, S., Boyacioglu, D., Toydemir, G. \& Capanoglu, E. (2014) Effect of industrial juice concentrate processing on phenolic profile and antioxidant capacity of black carrots. International Journal of Food Science and Technology, 49, 819-829.

Toydemir, G., Capanoglu, E., Gomez Roldan, M.V. et al. (2013). Industrial processing effects on phenolic compounds in sour cherry (Prunus cerasus L.) fruit. Food Research International, 53, 218-225.

Turfan, O., Turkyılmaz, M., Yemis, O. \& Ozkan, M. (2011). Anthocyanin and color changes during processing of pomegranate (Punica granatum L. cv. Hicaznar) juice from sacs and whole fruit. Food Chemistry, 129, 1644-1651.

Turfan, O., Turkyılmaz, M., Yemis, O. \& Ozkan, M. (2012). Effects of clarification and storage on anthocyanins and color of pomegranate juice concentrates. Journal of Food Quality, 35, 272-282.

Viuda-Martos, M., Navajas, Y.R., Lopez, J.F., Sendra, E., Barbera, E.S. \& Alvarez, J.A.P. (2010). Pomegranate and its many functional components as related to human health: a review. Comprehensive Reviews in Food Science and Food Safety, 9, 635-654.

Viuda-Martos, M., Navajas, Y.R., Lopez, J.F., Sendra, E., Barbera, E.S. \& Alvarez, J.A.P. (2011). Antioxidant properties of pomegranate (Punica granatum L.) bagasses obtained as co product in the juice extraction. Food Research International, 44, $1217-1223$.

Wootton-Beard, P.C., Moran, A. \& Ryan, L. (2011). Stability of the total antioxidant capacity and total polyphenol content of $23 \mathrm{com}-$ mercially available vegetable juices before and after in vitro digestion measured by FRAP, DPPH, ABTS and Folin Ciocalteu methods. Food Research International, 44, 217-222.

Xuan, L., Linwei, L., Wasila, H., Zhongmei, G., Tian, Y. \& Beita, Z. (2014). Variation of polyphenols composition and bioactivities of pomegranate wines along in vitro digestion process. Journal of Food and Nutrition Research, 2, 839-845. 